\title{
Comparison of Different Chromatic Dispersion Compensation Techniques in Radio over Fiber System
}

\author{
Jayasree Vadakke Kadangote, Neethu Johney, Asha Radhamany Somasekharan
}

Department of Electronics, Govt. Model Engineering College, Thrikkakara, Ernakulam, Kerala, India

\section{Email address:}

jaya@mec.ac.in (Jayasree V. K.), neethusjohney@gmail.com (Neethu J.), asharspillai@gmail.com (Asha R. S.)

\section{To cite this article:}

Jayasree Vadakke Kadangote, Neethu Johney, Asha Radhamany Somasekharan. Comparison of Different Chromatic Dispersion Compensation Techniques in Radio over Fiber System. American Journal of Optics and Photonics. Vol. 3, No. 2, 2015, pp. $24-29$. doi: 10.11648/j.ajop.20150302.11

\begin{abstract}
In this paper, we investigate the transmission of a $100 \mathrm{Gbit} / \mathrm{s}$ signal with $0.1 \mathrm{THz}$ carrier frequency using radio over fiber (RoF) for a fiber length of $50 \mathrm{~km}$. In our study, the impact of chromatic dispersion on the wireless signals transported through the fiber link and its mitigation using different dispersion compensation techniques are discussed. The techniques discussed here includes the optical single sideband transmission using dual electrode Mach Zehnder modulator, external filtering using FBG and compensation using chirped fiber grating. We also evaluated the performance of the three compensation techniques by plotting the eye diagram. The results clearly depict that compensation using chirped grating is the best among the three methods. The investigation is done on detailed simulative analysis using Optisystem.
\end{abstract}

Keywords: Radio Over Fiber (RoF), Chromatic Dispersion, Fiber Bragg Grating, Optical Single Sideband (OSSB), Chirped Fiber Bragg Grating (CFBG)

\section{Introduction}

Radio-over-Fiber (RoF) technology provides the base platform for the seamless integration of wireless and optical systems. It combines the technical advantages of the wireless and optical systems. Recently, radio-over-fiber (RoF) techniques have gained popularity because of the advantages it offers [1]. Some of the advantages of the RoF system are high bandwidth, low losses, mobility etc. RoF means the optical signal is being modulated at radio frequency and transmitted via the optical fiber. RoF improves the system flexibility and provides a very large coverage area without increasing the cost and complexity of the system [2][3].

In RoF systems, broadband microwave data signals are modulated onto an optical carrier at a central station (CS), and are then transported to base station (BS) using optical fiber. The RF signals are then transmitted by the basestations over small areas using microwave antennas i.e. the access link between the base station and the mobile user is the wireless link. This allows the base-stations to be extremely simple since they only need to contain optoelectronic conversion devices and amplifiers. The central station performs functions such as coding, modulation, multiplexing and up conversion. Centralization results in equipment sharing, dynamic resource allocation and more effective management [4]. All of this adds up to an access technology that makes life easier and cheaper for operators. The reason why RoF is able to shift system complexity away from the antenna is that optical fiber is an excellent low-loss and high bandwidth (50THz) transmission medium. Some of the advantages and benefits of the RoF technology compared with electronic signal distribution are large bandwidth, low attenuation loss, easy installation and maintenance, immunity to radio frequency interference, reduced power consumption, operation flexibility and dynamic resource allocation [5].

There are many methods for the generation and transmission of optical mm-wave in RoF systems. Some of the common optical mm-wave generation approaches are direct modulation [6], external optical modulation [7] [8], optical double-frequency heterodyning [9], Brillouin scattering [10], wavelength conversion [11], and continuum light filtering [12]. The simplest scheme among these is the direct modulation of a laser diode but it is limited by the laser chirp and a lower frequency response [13]. Even 
though optical heterodyning scheme has a great potential in producing high-frequency mm-wave, the quality of the mmwave produced by this technique depends on the coherence of the two laser lightwaves. Better signal performance at a high frequency can be generated by external modulation scheme [8][14][15]. External modulation applies to modulation schemes where the light source (laser) is driven by a constant-bias current that is higher than the threshold current. This bias current is usually optimized to get the best performance from the laser. Under this condition, the laser diode is said to be operating in $\mathrm{CW}$ mode, meaning that it emits constant light output. The constant bias eliminates issues such as laser chirp, saturation, clipping, and instability problems. This constant light is then modulated with an external optical modulator. This method requires only a simple configuration as the spectral components that are generated by external modulation are inherently phase-locked. Hence we can easily construct robust systems without using a complicated control mechanism. There are mainly two types of external modulators: Electro Absorption Modulators (EOM). EOMs are the most commonly used external modulators. The most widely used EOM is the Mach-Zehnder modulator, also referred to as the Mach-Zehnder interferometer. A MZM can be made of lithium niobate $\left(\mathrm{LiNbO}_{3}\right)$, gallium arsenide (GaAs), or indium phosphide (InP), materials that exhibit some anisotropy in their dielectric properties [17]. Among them Lithium Niobate $\left(\mathrm{LiNbO}_{3}\right)$ Mach-Zehnder (MZM) modulator is still considered as the device with the best performance [18].

In the external modulation schemes, the optical carrier is modulated by a radio signal to generate the sidebands at the transmitters, and the optical mm-wave consists of the optical carrier and its first-order sidebands. At the receiver, the beating between the optical carrier and the sidebands or the sidebands themselves can produce the required radio mm-waves. But when the optical carrier and the sidebands are transmitted over the optical fiber, due to the chromatic dispersion within the fiber, the two sidebands will experience different amounts of phase shift relative to the optical carrier. Upon detection at the photodetector, the square-law process generates two beat components at the desired mm-wave frequency. The received RF power of the mm-wave signal varies depending on the relative phase difference between the two beat components. The RF power variation is dependent on the fiber dispersion parameter, the transmission distance and also the mm-wave frequency. The fiber-induced dispersion penalties are so severe in directdetection optically-fed mm-wave systems. As a result various techniques have been proposed and demonstrated to overcome dispersion effects in such systems [19]. Amongst these techniques are the optical-single-sideband-withcarrier modulation scheme $(\mathrm{OSSB}+\mathrm{C})$, optical carrier suppression technique [20]-[22], external filtering [23][26], using chirped fiber gratings [23], using fiber nonlinearities [27]-[29], using DCF and using phase conjugation [30]. In this paper we discuss and compare the dispersion compensation techniques namely the external filtering using $\mathrm{FBG}, \mathrm{OSSB}+\mathrm{C}$ modulation using dual electrode Mach Zehnder modulator and chirped fiber bragg grating compensation.

\section{The Effect of Chromatic Dispersion in RoF Systems}

Even though there are many sources for signal impairments in the RoF link, the impact of fiber chromatic dispersion on the transported RF signals is the most dominant one. In RoF systems, the RF signal is used to directly modulate the laser diode in the central site (headend). The resulting intensity modulated optical signal consisting of the carrier and two sidebands (double-sideband (DSB) modulation) is then transported over the length of the fiber to the BS. At the optical receiver, each sideband beats with the optical carrier, thereby generating two beat signals which constructively interfere to produce a single component at the RF frequency. However, when the DSB signal is transmitted over fiber, chromatic dispersion causes each spectral component to experience different phase shifts depending on the link distance, modulation frequency, and the dispersion parameter of fiber. These phase shifts result in relative phase differences between the carrier and each sideband, and produce a phase difference in the two beat signals at the RF frequency, which results in a power degradation of the composite RF signal. When the phase difference is $\pi$, complete cancellation of the RF signal occurs. As the RF frequency increases, the effect of dispersion is even more pronounced and the fiber-link distance severely limited [25] [30]. The RF power variation is thus dependent on the fiber dispersion parameter, the transmission distance, and the mmwave frequency as governed by the Eq.1 [19]:

$$
P_{R F} \propto \cos ^{2}\left[\pi c L D\left(\frac{f_{m m}}{f_{0}}\right)^{2}\right]
$$

Where $D$ represents the fiber dispersion parameter in $\mathrm{ps} / \mathrm{nm} / \mathrm{km}, c$ is the velocity of light in a vacuum, $L$ is the fiber transmission length, $f_{\mathrm{mm}}$ represents the mm-wave modulating frequency, and $\mathrm{f}_{0}$ is the optical carrier center frequency [19].

Since the fiber-induced dispersion penalties are so severe in direct-detection optically fed mm-wave systems, various techniques have been devised and proposed to overcome the dispersion effects in such systems. A convenient technique to overcome the fiber dispersion effect is by simply removing one of the optical sidebands in an optical DSB modulated signal. J. Park, W. V. Sorin, and K. Y. Lau [29] proposed a simple method for the elimination of the fiber dispersion penalty on conventional external optical intensity modulation at millimeter-waves using a fiber Bragg grating filter to produce single-sideband optical modulation. G.H Smith, D. Novak, and Z. Ahmed [25] proposed another method for generating an optical carrier with single sideband modulation using a dual-electrode Mach-Zehnder modulator biased at quadrature so as to reduce the effect of chromatic dispersion. 
The optical carrier suppression scheme is another effective method to combat dispersion effects in mm-wave fiberwireless links. J.M. Fuster, J. Marti, and J. L. Corral [27] proposed that biasing a dual-electrode Mach-Zehnder modulator (MZM) at the minimum transmission point of the transfer function, the optical carrier will be suppressed and a double-sideband-suppressed carrier optical signal will be generated. Such an implementation requires only half the desired modulating frequency to drive the MZM. The mixing of the two optical carriers in a high-speed photodetector generates a single beat component at twice the drive frequency, which is not affected by dispersion-induced RF penalties.

In addition to the external modulation techniques, tapered linearly chirped fiber gratings have also been used to counter dispersion in mm-wave fiber links. J Marti, J. M. Fuster, and R. I. Laming [31] demonstrated experimentally that the dramatic microwave power degradation that appears in conventional $1550 \mathrm{~nm}$ lightwave amplitude modulated systems due to chromatic dispersion can be overcome by using a tapered linearly chirped fiber grating.

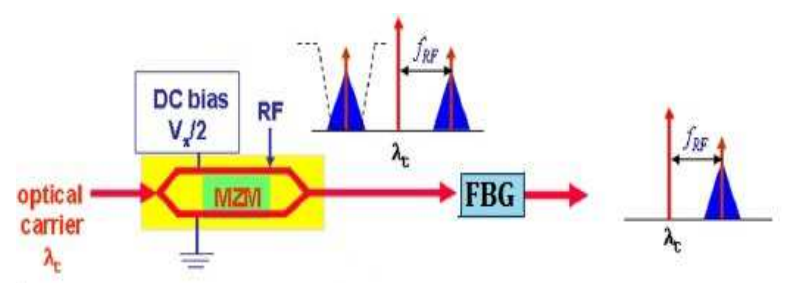

Fig. 1. $O S S B+C$ signal generation using $F B G$.

\section{Dispersion Compensation Techniques}

As mentioned above, different techniques can be used for the compensation of chromatic dispersion. In this paper we mainly discuss three techniques namely the external filtering using FBG, OSSB $+\mathrm{C}$ modulation using dual electrode Mach Zehnder modulator and chirped fiber bragg grating compensation. Principles of these techniques are discussed below.

One of the simplest method to overcome the impact of fiber chromatic dispersion on mm-wave modulated optical signals is by using the OSSB $+\mathrm{C}$ modulation format. The formatted signal can be generated via cancellation of the unwanted optical sideband within an external optical modulator or using an external filter.

The effect of chromatic dispersion can be eliminated using a fiber Bragg grating which act as a narrow band notch filter and removes the unwanted sideband thereby producing $\mathrm{OSSB}+\mathrm{C}$ modulation. This is a simple method for the elimination of the fiber dispersion penalty on conventional external optical intensity modulation at millimeter-waves. The method is depicted in figure 1. Although this technique is simple to implement, the limited flexibility makes the implementation difficult to accommodate for modifying the mm-wave frequency.



Fig. 2. OSSB $+C$ signal generation using DEMZM.
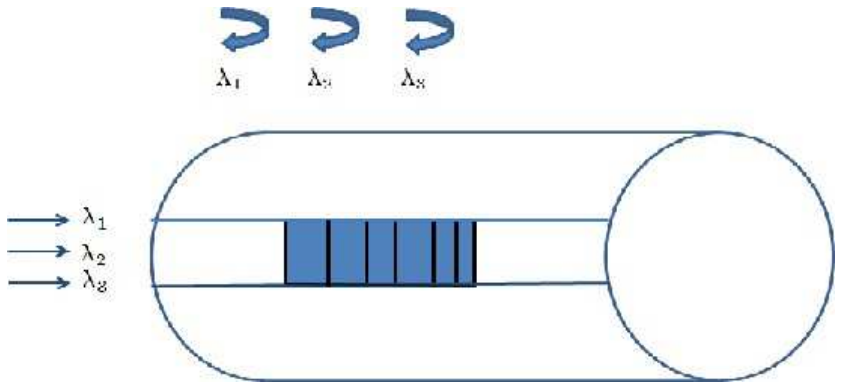

Fig. 3. Chromatic dispersion mitigation method using chirped grating.

Another method for generating an optical carrier with single sideband modulation using a dual-electrode MachZehnder modulator biased at quadrature so as to reduce the effect of chromatic dispersion. The method is shown in figure 2. When the dual electrode Mach-Zehnder modulator (DEMZM) is biased at quadrature and when the RF signal is applied to both electrodes with a $90^{\circ}$ phase shift between the two electrodes, the interaction between the RF modulation and the optical signals results in the suppression of one of the odd-harmonics' modulation sidebands. This results in the generation of optical single sideband with carrier $(\mathrm{OSSB}+\mathrm{C})$ modulation.

In addition to the external modulation techniques, tapered linearly chirped fiber gratings have also been used to counter dispersion in mm-wave fiber links. Figure 3 depicts the block diagram of the above mentioned experimental set up. A specially designed tapered linearly chirped fiber grating is inserted at the output of the fiber via an optical coupler to overcome the dispersion.

Linear chirped grating allows creating a time delay between different spectral components of the signal. For example, in SMF at $1.55 \mu \mathrm{m}$ group velocity dispersion creates a negative chirp of the pulses, which means that the higher frequencies (which propagate faster) are in the leading part of the pulse and the lower (propagating slower) in the trailing one. Because of this different velocity of propagation of different spectral components, the pulse spreads. If we create fiber grating with period linearly reducing along the grating, because of the fact that the higher frequencies will reflect after longer propagation in the grating, a time delay between lower and higher frequency components will appear which is just opposite to this created in the SMF. Therefore propagating and reflecting our pulse in this device will allow compensating the dispersion broadening of our pulse. 
Table 1. Simulation Parameters.

\begin{tabular}{lll}
\hline Sl. No. & Parameters & Value \\
\hline 1 & Bit rate & $100 \mathrm{Gbps}$ \\
2 & Frequency of sine generator & $0.1 \mathrm{THz}$ \\
3 & Frequency of CW laser & $193.1 \mathrm{THz}$ \\
4 & Power of CW laser & $0 \mathrm{dBm}$ \\
5 & Half wave switching voltage $\mathrm{V}_{\pi}$ of $\mathrm{LiNbO}_{3}$ & $4 \mathrm{~V}$ \\
6 & MZM & $20 \mathrm{~dB}$ \\
7 & Extinction ratio of LiNbO $\mathrm{MZM}_{3}$ & $5 \mathrm{~dB}$ \\
8 & Insertion loss of LiNbO $\mathrm{MZM}_{3}$ & $4 \mathrm{~V}$ \\
9 & Switching RF voltage of $\mathrm{LiNbO}_{3} \mathrm{MZM}^{2}$ & $16 \mathrm{ps} / \mathrm{ns} / \mathrm{km}$ \\
10 & Fiber dispersion & $1 \mathrm{~A} / \mathrm{W}$ \\
\hline
\end{tabular}

\section{System Description and Simulation Details}

An optical communication system involving external modulation as shown in Figure (4) have been simulated using Optisystem 7 so as to investigate the effect of chromatic dispersion on system performance.

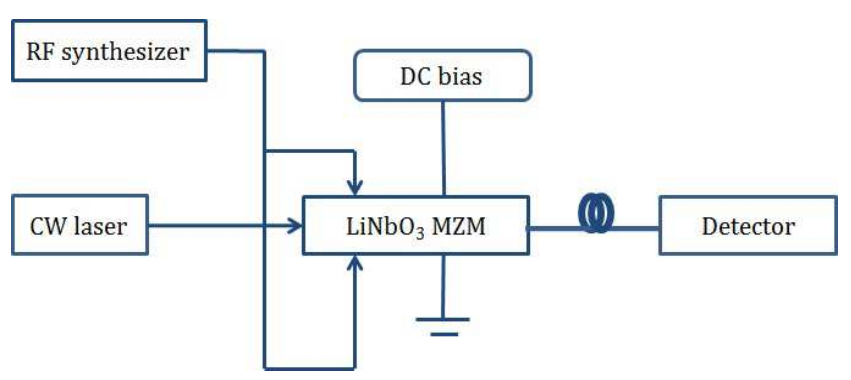

Fig. 4. Optical communication system.

Here, the system being considered is a radio over fiber optical communication system. The $\mathrm{LiNbO}_{3} \mathrm{MZM}$ is driven by an RF signal of frequency $f_{R F}$ which is equal to $0.1 \mathrm{THz}$. The CW laser source is taken with power of $0 \mathrm{dbm}$ and center emission frequency $193.1 \mathrm{THz}$. The half wave switching voltage $\mathrm{V}_{\pi}$ of $\mathrm{LiNbO}_{3} \mathrm{MZM}$ is $4 \mathrm{~V}$. After being modulated at the external modulator, the intensity modulated optical carrier will be transmitted through the optical fiber and detected by the photodetector. We use the parameters in Table 1 in mandate to simulate the system.

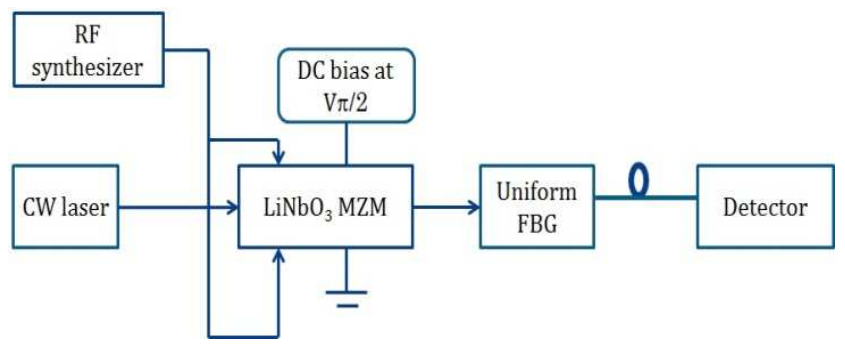

Fig. 5. Dispersion compensation using FBG.

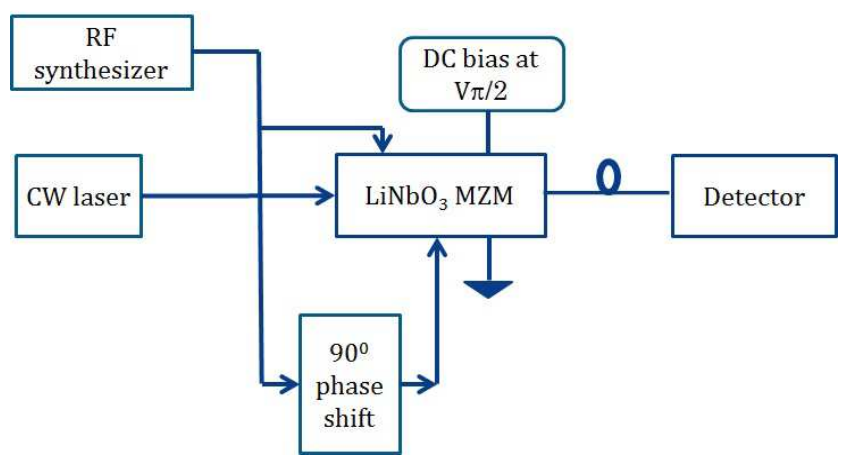

Fig. 6. Dispersion compensation using DEMZM.

Another technique uses dual electrode Mach-Zehnder modulator (DEMZM) biased at quadrature point to produce optical single sideband with carrier. This compensation scheme is depicted in figure (6).

Third technique that is used to counter dispersion in mmwave fiber links is using linearly chirped fiber bragg gratings. This method is depicted in the figure (7).

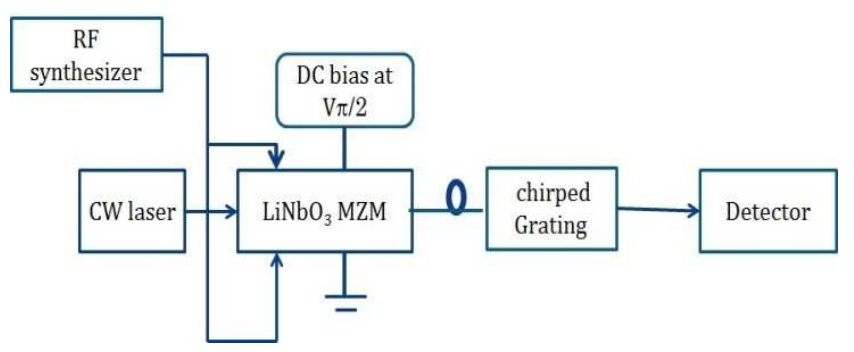

Fig. 7. Dispersion Compensation using CFBG.

\section{Results and Discussions}

In optical communication systems, only optical signal to noise ratio (OSNR) could not accurately measure the system performance. The eye diagram is a common indicator of performance in digital transmission systems. The eye diagram is an oscilloscope display of a digital signal, repetitively sampled to get a good representation of its behavior.

When the intensity modulated optical carrier along with the two sidebands is transmitted through the dispersive fiber, RF power fading occurs. As a result the eye diagram obtained will be distorted as shown in Figure 8(a).

But when dispersion compensation is provided better eye diagram is obtained which indicates that dispersion has been reduced.

The eye diagrams for the three compensation schemes are shown in Figure $8 \mathrm{~b}, 8 \mathrm{c}$ and $8 \mathrm{~d}$. It is clear from the figure that the compensation using CFBG yields a better performance compared to the other two techniques. Optical single sideband with the carrier obtained using DEMZM and FBG are shown in figure 9. 


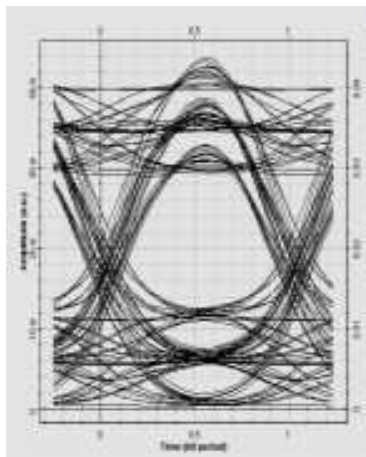

(a)

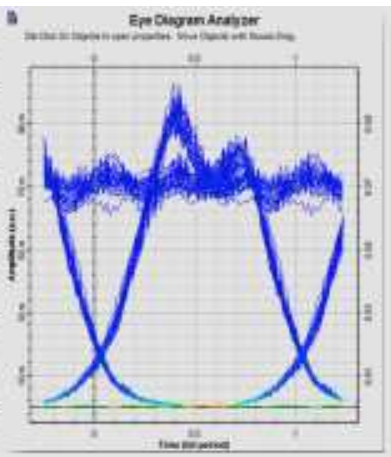

(b)

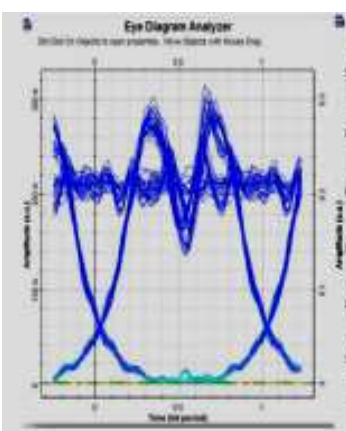

(c)

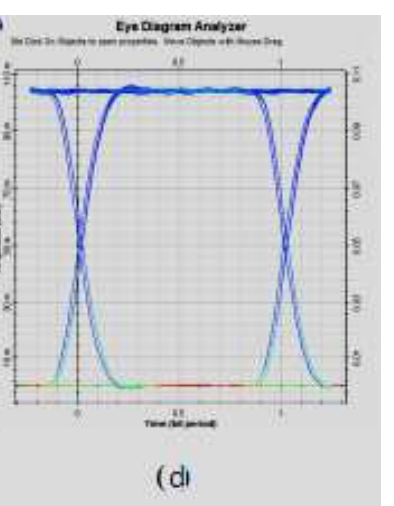

(d)

Fig. 8. Eye diagram obtained (a) without compensation (b) with compensation using FBG (c) with DEMZM (b) with CFBG.

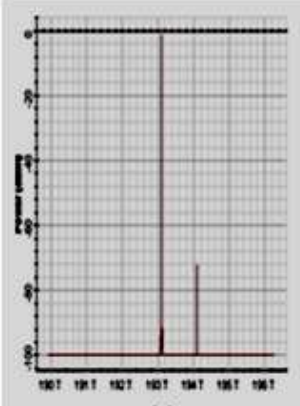

(a)

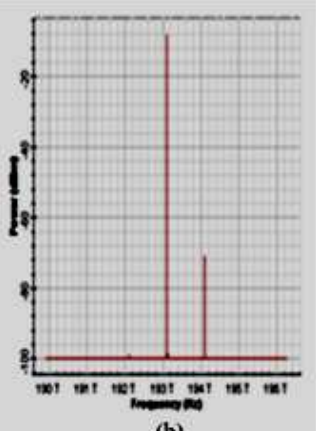

(b)
Fig. 9. Optical single sideband signal with carrier obtained using (a) $D E M Z M(b) F B G$

\section{Conclusion}

In this paper, we have discussed the effect of chromatic dispersion on the transmission of intensity modulated optical carrier through the fiber link in a RoF system. We have also discussed and simulated dispersion compensation technique using $\mathrm{FBG}, \mathrm{OSSB}+\mathrm{C}$ modulation using dual electrode Mach Zehnder modulator and chirped fiber bragg grating. The three compensation techniques were compared by plotting eye diagram. It is found that the compensation using chirped fiber bragg grating yields a better performance compared to the other two schemes.

\section{References}

[1] H. Al-Raweshidy, "Radio over fibre technology for the next generation" in Radio over Fiber Technologies for Mobile Communications Networks", Artech House, Inc. USA, 2002.

[2] Xavier N. Fernando, "Radio over fiber for wireless communications from fundamentals to advanced topics", Wiley publications, $1^{\text {st }}$ edition, 2014.

[3] Naresh Kumar, AmitGarg and SandeepPanwar, " A review paper on radio over fibre technology" International Journal of Applied Engineering Research, ISSN 0973-4562 vol.7, no.11, pp. 167-170,2012.
[4] Ng'oma, A. and Sauer, M., "Radio-over-Fiber technologies for high data rate wireless applications," Proc of Sarnoff Symposium, 2009.

[5] Anthony Ng'oma," Radio-over -Fiber Technology for Broadband Wirless Communication Systems", Ph.D Thesis, 2005

[6] G. Maury, A. Hilt, T. Berceli, B. Cabon, and A. Vilcot, "Microwave frequency conversion methods by optical interferometer and photodiode," IEEE Trans. Microw. Theory Tech., vol. 45, no. 8, pp. 1481-1485, Aug. 1997

[7] J. Kim, et.al, "Development and RF characteristics of analog $60-\mathrm{GHz}$ electroabsorption modulator module for RF/optic conversion," IEEE Trans. Microw. Theory Tech., vol. 54, no. 2, pp. 780-787, Feb. 2006.

[8] Jianxin Ma, J. Yu, Chongxiu Yu, XiangjunXin, Junying Zeng, and L. Chen, "Fiber dispersion influence on transmission of the optical millimeter-waves generated using ln-mzm intensity modulation", journal of lightwave technology, vol. 25, no. 11, pp.3244-3256, Nov. 2007

[9] Z. Deng and J. Yao, "Photonic generation of microwave signal using a rational harmonic mode-locked fiber ring laser," IEEE Trans. Microw.Theory Tech., vol. 54, no. 2, pp. 763-767, Feb. 2006.

[10] Y. Shen, X. Zhang, and K. Chen, "Optical single sideband modulation of 11-GHz ROF system using stimulated Brillouin scattering," IEEE Photon.Technol.Lett., vol. 17, no. 6, pp. 1277-1279, Jun. 2005.

[11] J. Yu, et.al., "Seamless integration of an $8 \times 2.5 \mathrm{~Gb} / \mathrm{s}$ WDMPON and radio-over-fiber using all-optical up conversion based on Raman-assisted FWM," IEEE Photon. Technol. Lett., vol. 17, no. 9, pp. 1986-1988, Sep. 2005.

[12] T. Nakasyotani, H. Toda, T. Kuri, and K.Kitayama, "Wavelengthdivision- multiplexed millimeter-waveband radio on-fiber system using a supercontinuum light source," $J$. Lightw. Technol., vol. 24, no. 1, pp. 404-410, Jan. 2006

[13] A. Kaszubowska, L. P. Barry, and P. Anandarajah, "Effects of intermodulation distortion on the performance of a hybrid radio/fiber system employing a self-pulsating laser diode transmitter," IEEE Photon. Technol.Lett., vol. 15, no. 6, pp. 852-854, Jun. 2003. 
[14] K. Noguchi, O. Mitomi, and H. Miyazawa, "Millimeter-wave Ti:LiNbO3 optical modulators," J. Lightw. Technol., vol. 16, no. 4, pp. 615-619, Apr. 1998.

[15] J. C. Cartledge, "Performance of $10 \mathrm{~Gb} / \mathrm{s}$ lightwave systems based on lithium niobate Mach-Zehnder modulators with asymmetric Y-branch waveguides," IEEE Photon. Technol. Lett., vol. 7, no. 9, pp. 1090-1092, Sep. 1995.

[16] Yejun Fu, Xiupu Zhan, et.al,"Mach-Zehnder", IEEE microwave magazine, pp. 102- 107,November 15,2013.

[17] G. L. Li and P. K. L. Yu, "Optical intensity modulators for digital and analog applications," J. Lightwave Technol., vol. 21, no. 9, pp. 2010-2030, 2003.

[18] http://www.photline.com

[19] Christina Lim, et.al,"Mitigation strategy for transmission impairments in millimeterwave radio-over-fiber networks" Journal of Lightwave Technology, Vol. 28, No. 4, Feb. 15, pp. 201-211,2010

[20] M. J. LaGasse, W. Charczenko, M. C. Hamilton, and S. Thaniyavarn, "Optical carrier filtering for high dynamic range fibre optic links," Electron. Lett. 30, pp.2157-2158 (1994).

[21] R. D. Esman and K. J. Williams, "Wideband efficiency improvement of fiber optic systems by carrier substraction," IEEE Photon. Technol. Lett. 7, pp.218-220 (1995).

[22] H. Toda, et.al "25-GHz channel spacing DWDM multiplexing using an arrayed waveguide grating for $60-\mathrm{GHz}$ band radioon-fiber systems," International Topical Meeting on Microwave Photonics (IEEE, 2003), pp. 287-290.

[23] G. J. Meslener, "Chromatic dispersion induced distortion of modulated monochromatic light employing direct detection," IEEE J. Quantum Electron. QE-20, pp. 1208-1216 (1984).

[24] H. Schmuck, "Comparison of optical millimeter-wave system concepts with regard to chromatic dispersion," Electron. Lett. 31,pp. 1848-1849 (1995).

[25] G. H. Smith,et.al, "Technique for optical SSB generation to overcome dispersion penalties in fibre-radio systems," Electron. Lett. 33, pp.74-75 (1997).

[26] R. A. Griffin,et.al, "Dispersion-tolerant subcarrier data modulation of optical millimetre-wave signals," Electron. Lett. 32 , pp.2258-2260 (1996).

[27] J. M. Fuster, et.al, "Chromatic dispersion effects in electrooptical upconverted millimetre-wave fibre optic links," Electron. Lett. 33,pp. 1969-1970 (1997).

[28] Z. Jia, J. Yu, and G.-K. Chang, "A full-duplex radio-over-fiber system based on optical carrier suppression and reuse," IEEE Photon. Technol. Lett. 18, pp.1726-1728 (2006).

[29] J. Park,et.al, "Elimination of the fibre chromatic dispersion penalty on $1550 \mathrm{~nm}$ millimetre-wave optical transmission," Electron. Lett. 33, pp. 512-513 (1997).

[30] K. Yonenaga and N. Takachio, "A fiber chromatic dispersion compensation technique with an optical SSB transmission in optical homodyne detection systems," IEEE Photon. Technol. Lett. 5, pp.949-951 (1993).

[31] J Marti, et.al , "Experimental reduction of chromatic dispersion effects in lightwave microwave/millimetre-wave transmissions using tapered linearly chirped fibre gratings," Electron. Lett., vol. 33, pp. 1170-1171, 1997. 